\title{
Characterization of Bruton's Tyrosine Kinase Gene and Protein from Marine Sponge Suberites domuncula ${ }^{\dagger}$
}

\author{
Drago Perina, Andreja Mikoč, Matija Harcet, Mirna Imešek, \\ Dujo Sladojević, Ana Brcko, and Helena Ćetković*
}

Division of Molecular Biology, Rudjer Boskovic Institute, P.O. Box 170, HR-10002 Zagreb, Croatia

RECEIVED DECEMBER 23, 2010; REVISED JUNE 23, 2011; ACCEPTED MAY 8, 2012

\begin{abstract}
Bruton's tyrosine kinase (Btk) is a member of Tec family of protein tyrosine kinases. It is expressed in B cells and myeloid cells in humans and it is crucial for maturation of B-lineage lymphoid cells. Mutations in the gene encoding human Btk result in hereditary immunodeficiency X-linked agammaglobulinemia. Sponge proteins can probably reflect characteristics of the ancestral metazoan homologues before their diversifications and specializations in complex animals.

Gene coding for sponge Btk-like protein was fully characterized and its structure was compared with homologous genes from other organisms. Kinase activity of sponge protein was compared to human Btk activity.

We showed that sponge Btk-like gene is probably the most ancient member of Tec family while other members arose from the ancestral gene present in chordate ancestor. The ancestral-type protein was structurally and functionally similar to the highly sophisticated enzyme it is today, whose functions are usually associated with higher Metazoa. (doi: 10.5562/cca1862)
\end{abstract}

Keywords: Porifera, Btk, Tec family PTK, gene structure

\section{INTRODUCTION}

The family of protein tyrosine kinases (PTKs) is a large group of proteins which have evolved together with multicellularity and are found in animals (Metazoa) and choanoflagellates. ${ }^{1}$ PTKs have evolved in step with the increasing complexity of multicellular organisms and over a hundred PTKs are encoded in the human genome. ${ }^{2}$ PTKs from the Tec family are non-receptor, cytoplasmic kinases. This family consists of five mammalian members: Btk, Bmx, Itk, Tec and Txk kinases. The evolution of PTKs from the Tec family has been described earlier. ${ }^{1}$ The ancestral Tec PTK was present in unicellular eukaryotes prior to the appearance of metazoans. Tec family PTK is present in Monosiga brevicolis, a fully sequenced representative of choanoflagellates, which are the closest relatives to metazoans. ${ }^{1}$ Tec PTKs are absent from the genome of Caenorhabditis elegans. ${ }^{3}$ Btk PTK contains five domains from the $N$-terminus: the Pleckstrin homology (PH) domain, followed by the Tec homology (TH) domain, which is further subdivided into the $\mathrm{Zn}^{2+}$-binding Btk motif and a proline-rich region, the Src homology 3 and 2 (SH2 and
SH3) and finally the tyrosine kinase (TK) catalytic domain. ${ }^{4}$ Human Btk is expressed in all hematopoietic cells except T-lymphocytes and plasma cells ${ }^{4,5}$ and has a well-defined role in B cell antigen receptor (BCR) signaling. ${ }^{6}$ Mutations in Btk gene cause $\mathrm{X}$-linked agammaglobulinemia (XLA). XLA is a severe hereditary immune disease characterized by a lack of peripheral B cell and low levels of serum Ig. ${ }^{7}$ In mouse, btk mutations or deletion cause $\mathrm{X}$-linked immunodeficiency (Xid), with a deficit of B2 B cells, the absence of B1 B cells and low levels of serum Ig. ${ }^{8}$

Sponges (Porifera) are excellent model organisms for molecular evolutionary studies. They represent the most basal metazoan phylum which existed prior to the Cambrian explosion and have changed little since, ${ }^{9}$ and can therefore be considered as living fossils. They do not have true tissues and organs, and lead a sessile lifestyle. In many aspects of their molecular biology, such as gene content, their functional repertoire and intron positions, sponges such as Suberites domuncula and Amphimedon queenslandica (with large transcriptome or genome datasets available) probably reflect the situation in metazoan ancestor (Urmetazoa). Systematic

\footnotetext{
$\dagger$ Presented at the $10^{\text {th }}$ Congress of the Croatian Society of Biochemistry and Molecular Biology held in Opatija, Croatia, September 15-18, 2010.

* Author to whom correspondence should be addressed. (E-mail: cetkovic@irb.hr)
} 
analysis of the sponge gene repertoire showed that the genomic complexity was already present at the very beginning of metazoan evolution, before the appearance of tissue or any other complex morphological feature found in extant animals. ${ }^{10}$ Previous analyses of phylogenetically conserved genes/proteins in sponges identified a number of very sophisticated multifunctional enzymes whose functions are usually associated with human diseases. ${ }^{3,11,12}$

Btk-like 700-aa-long protein from the marine sponge Suberites domuncula (Demospongia) was described previously. ${ }^{3}$ Sponge Btk-like protein contains all of the characteristic domains for the Tec family of PTKs. The highest homology (38\% identity, $55 \%$ overall similarity) was found with human Btk PTK and therefore the sponge homolog was named BtkSD. Our finding showed that Tec family gene/protein already existed in the common metazoan ancestor more than 600 millions years ago, i.e. before the separation of sponges from other animal lineages and remained highly conserved during animal evolution. ${ }^{3}$ Our goal was to determine the structure of the $b t k S D$ gene, compare it with its metazoan homologs and test whether the sponge BtkSD protein has a similar level of kinase activity as its human homolog.

\section{EXPERIMENTAL}

\section{Materials}

Live specimens of the sponge Suberites domuncula (Porifera, Demospongia, Tetractinomorpha, Hadromerida, Suberitidae) were collected in the northern Adriatic Sea near Rovinj, Croatia, and stored at $-80^{\circ} \mathrm{C}$.

\section{Sponge btkSD Gene}

For genomic DNA preparation, the specimens of $S$. domuncula were cut into pieces, frozen in liquid nitrogen, ground to fine powder from which total DNA was isolated using the Genomic DNA Purification kit (QIAGEN), according to the manufacturer's instructions. Sponge $b t k S D$ gene was amplified, using KOD XL polymerase (Novagen). Three sets of specific primers were used to amplify whole btkSD gene: (5'-TTTCTGCTGGGAACAGGAT-3' and 5'-GTGGGGAGGGTGCGAGGTT-3'); (5'-GAGGCAGTGTGAAAGGTTATGTTG-3' and 5'-TTGCTCATCTCGTGCTCGCC-3'); (5'-CCCCGGAGCCCAAACCAT-3' and 5'AAATGGGTTACATCCACACACA-3'). The amplified fragments were separated on a $0.8 \%$ agarose gel, purified and sequenced (ABI PRISM ${ }^{\mathbb{R}}$ 3100-Avant Genetic Analyzer, Applied Biosystems, USA) using ABI PRISM BigDye Terminator v.3.1 Ready Reaction Cycle Sequencing Kit.

The reverse specific primer 5'- GGCTTGGTTTCCTGGCATCTTATAGTGAG-3' was used in conjunc- tion with the vector specific primer $\mathrm{T} 3 / \mathrm{T} 7$ to amplify the 5'-promoter region of the btkSD gene on the genomic library from $S$. domuncula. ${ }^{13}$

Nucleotide sequences were analyzed using Lasergene sequence analysis software (DNAStar, Madison, WI). Homologous sequences from other organisms were identified by BLAST and retrieved from NCBI's GenBank. Multiple sequence alignments (MSA) and construction of the phylogenetic tree from the MSA was performed with ClustalX program. ${ }^{14}$ The exact position and the phase of each intron was verified by manual inspection.

The putative transcription factor binding sites in promoters of $b t k$ genes were identified using TFSEARCH (http://www.cbrc.jp/research/db/TFSEARCH.html), and there searching within TRANSFAC database. ${ }^{15}$ "Hamming Clustering Method for TATA signal prediction in Eukaryotic Genes" was used for TATA-box identification. ${ }^{16}$

A sample of 224 coding sequences of $S$. domuncula (including cDNA coding BtkSD) was collected for analysis of synonymous codon usage. ${ }^{17}$ CODONW program was used for synonymous codon usage analysis (http://codonw.sourceforge.net/) and calculated GC3S, Fgc and Fop parameters. GC3S is fraction of codons in gene which have either a guanine or cytosine at third codon position. Fgc is $\mathrm{G}+\mathrm{C}$ content of gene. Fop is the frequency of optimal codons used in gene, the ratio of optimal codons to synonymous codons. This value is always between 0 , which indicates no usage of optimal codon, and 1 which indicates that only optimal codons are used.

\section{Plasmid Constructions}

The cDNA for $\mathrm{BtkSD}^{3}$ was recloned using PCR into BamHI (5'-GACAGGATCCCAGTGGTTAGTCATC-3') and NdeI (5'-CACTATCATATGCCAGGAAACCAAGCC-3') restriction sites of $\mathrm{pET} 15 \mathrm{~b}$ expression vector (Novagen) downstream from the thrombin cleavage site.

\section{Protein Expression, Purification and Western Analysis}

BtkSD was overproduced in Escherichia coli strain BL21 tagged with six histidine residues at the $\mathrm{N}$-terminus and purified to homogeneity from bacterial lysates using cobalt affinity chromatography (TALON). Soluble protein visible after SDS-PAGE was obtained under modified growth conditions: $E$. coli strain BL21 harboring the plasmid construct was grown to $\mathrm{OD}_{600} 1.5$ and induced with $0.05 \mathrm{mM}$ IPTG for $3 \mathrm{~h}$ at $18{ }^{\circ} \mathrm{C}$. Cells were incubated 30 minutes on ice in lysis buffer (50 mM Tris $\mathrm{HCl}, 300 \mathrm{mM} \mathrm{NaCl}, 10 \mathrm{mM}$ imidazole, and $1 \mathrm{mg} / \mathrm{mL}$ lysozyme $)$ and sonicated $8 \times 30 \mathrm{sec}(50 \%$ of the full power). After centrifugation (12000 rpm) for $25 \mathrm{~min}$ at $4{ }^{\circ} \mathrm{C}$, the supernatant was applied onto 
cobalt-charged agarose column (TALON). Histidine tagged proteins were eluted with $500 \mathrm{mM}$ imidazole. The samples were loaded on SDS-PAGE and electrotransfered to a PVDF Hybond-P membrane (Amersham Biosciences). The membranes were incubated with anti-His antibody (Amersham Biosciences) for detection of 6 His-BtkSD. Protein bands were visualized using chemiluminiscence detection (Amersham ECL Plus, GE Healthcare).

BtkSD protein was applied to a PD-10 desalting gel filtration column (Sephadex, GE Healthcare) equilibrated and eluted with $20 \mathrm{mM}$ Tris $\mathrm{HCl}, \mathrm{pH}=9$ (start buffer). Ion-exchange chromatography (monoQ 5/50 GL column) using the fast protein liquid chromatography (FPLC) system was employed for protein purification. The column was equilibrated with start buffer and eluted with linear $\mathrm{NaCl}$ gradient up to $1.0 \mathrm{~mol} \mathrm{dm}{ }^{-3}$. Amicom Ultra-15 was used for desalting and buffer exchange (25 mM HEPES, $\mathrm{pH}=7.5,25 \mathrm{mM} \mathrm{NaCl}, 10 \mathrm{mM}$ $\mathrm{KCl}, 10 \mathrm{mM} \mathrm{MgCl}_{2}, 2 \mathrm{mM}$ DTT, $0.008 \%$ triton X-100).

\section{Kinase Assay}

Kinase activity was measured using a coupled pyruvate kinase-lactate dehydrogenase assay. Reaction kinetics was followed by connecting the turnover of ATP to the turnover of NADH to NAD. NADH concentrations were measured by spectrophotometry. Five hundred $\mu \mathrm{L}$ of reaction mixtures were incubated in quartz cuvettes at room temperature in the presence of ATP as phosphate donor and poly EY (4:1) MW 20000-50000 as substrate. The final concentrations were as follows: $25 \mathrm{mM}$ HEPES, $\mathrm{pH}=7.5,25 \mathrm{mM} \mathrm{NaCl}, 10 \mathrm{mM} \mathrm{KCl}, 10 \mathrm{mM}$ $\mathrm{MgCl}_{2}, 2 \mathrm{mM}$ phosphoenolpyruvate, $0.25 \mathrm{mM} \mathrm{NADH}$, $1 \mathrm{mM}$ ATP, $2 \mathrm{mM}$ DTT, 2 units of pyruvate kinase, 3 units of lactate dehydrogenase, $0.008 \%$ triton X-100 and $0.2 \mathrm{mg} / \mathrm{mL}$ poly EY. Reactions were initiated by the addition of $25 \mathrm{nM}$ of BtkSD enzyme and activity was monitored in an Ultrospec ${ }^{\circledR} 2100$ pro (Amersham Pharmacia Biotech, USA) measuring the decrease in absorbance at $\lambda=340 \mathrm{~nm}$. The control reactions omitting BtkSD produced minor rates but were subtracted from the BtkSD values. Reactions were done in triplicate.

\section{RESULTS AND DISCUSSION}

\section{Phylogenetic Analysis}

It is already known that Tec family PTK is present in the unicellular choanoflagellate Monosiga brevicollis which is the closest known relative to metazoans. ${ }^{1}$ In invertebrate and some vertebrate (amphibian) genomes, a single gene from the Tec family PTK was found which showed the highest similarity to Btk. ${ }^{1}$ Interestingly, we found two different Tec family PTKs from Monosiga ovata (BAG55524; BAG55489) which share

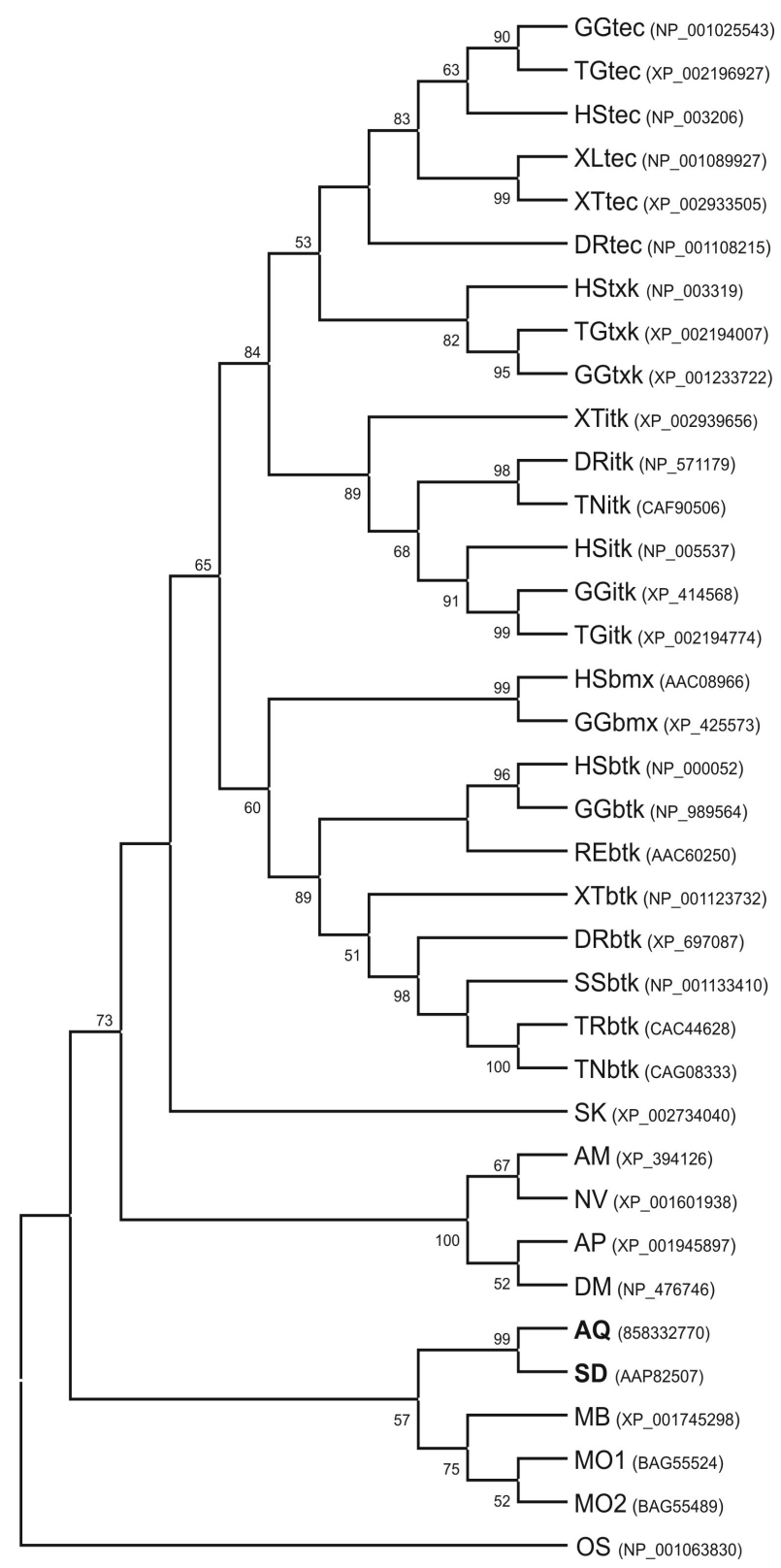

Figure 1. Maximum parsimony phylogenetic tree of Tec family of PTKs. Proteins from the following species were analysed:, Suberites domuncula (SD), Amphimedon queenslandica (AQ), Monosiga brevicollis (MB), Monosiga ovata (MO), Drosophila melanogaster (DM), Acyrthosiphon pisum (AP), Nasonia vitripennis (NV), Apis mellifera (AM), Saccoglossus kowalevskii (SK), Tetraodon nigroviridis (TN), Takifugu rubripes (TR), Salmo salar (SS), Danio rerio (DR), Xenopus (Silurana) tropicalis (XT), Gallus gallus (GG), Homo sapiens (HS), Xenopus laevis (XL), Taeniopygia guttata (TG), Oryza sativa (OS).

$65 \%$ similarity. Both of them showed the highest similarity with Btk Tec family member which is indicative for independent duplication not related to the appearance of recent Tec family members. Furthermore, we found three different Tec family PTKs from 
Xenopus tropicalis (XP_002933505, XP_002939656, NP_001123732), whereas there is only one present in Xenopus laevis. This is probably due to the lack of complete genome sequence of $X$. laevis rather than difference in number of Tec family members in those two closely related species. Phylogenetic analysis of TK domains PTKs from the Tec family was presented in Figure 1. The Btk-like homologs from sponges clearly group together with those from choanoflagellates. Sponge Btk-like protein reflects the characteristics of the protein ancestral to Tec family, before the duplications and diversifications within the metazoan lineage.

\section{Analysis of btkSD Gene}

The btkSD gene from $S$. domuncula encompasses one intron in 5' untranslated region (5'UTR; $222 \mathrm{bp}$ ) and 15 introns in the coding region ( $2500 \mathrm{bp}, 522 \mathrm{bp}, 324 \mathrm{bp}$, 246 bp, 432 bp, 72 bp, 79 bp, 229 bp, 223 bp, 164 bp, $71 \mathrm{bp}, 283 \mathrm{bp}, 187 \mathrm{bp}, 253 \mathrm{bp}$ and $255 \mathrm{bp}$ ) with the average length of $378 \mathrm{bp}$. Introns in human btk gene are much longer ranging between 179 and 10747 bp with the average length of $1898 \mathrm{bp}$. Sponge introns are relatively short with the exception of the first intron in coding region. The same was found for introns in several other sponge genes. ${ }^{18,19}$ While intron lengths drastically differ between these two organisms, exon lengths are pretty much similar. Human coding exons are ranging from 63 to $217 \mathrm{bp}$ in size with the average length of 110 bp. Sponge coding exons range from 55 to $389 \mathrm{bp}$ in size with the average length of $131 \mathrm{bp}$.

Multiple sequence alignment of $b t k S D$ gene homologues was produced and used to compare intron positions (Figure 2). The sponge $b t k S D$ gene has three introns in the $\mathrm{PH}$ domain, one intron at the end of $\mathrm{TH}$ domain (prolin rich region), one intron in the $\mathrm{SH} 3$ domain, one intron in front of the $\mathrm{SH} 2$ domain, two introns in $\mathrm{SH} 2$ domain and one in front of the TK domain.
Within the TK domain of $b t k S D$ gene there are six introns: one intron is in the following subdomains I, III, $\mathrm{V}$, Vib, IX and XI. The human homolog has 17 introns, D. melanogaster homolog has 11 introns and M. brevicollis homolog 10 introns. The $b t k S D$ gene from sponge has 9 introns in the same positions and the same phases as the human homolog and three more introns found in the same phases but on slightly different positions within the unconserved regions, probably of the same origin. On the other hand, $b t k S D$ gene from sponge has only three introns in the same positions and the same phases as the fruit fly homolog and two more introns probably of the same origin (in the same phases but slightly different positions). D. melanogaster probably lost seven ancestral introns and gained six new ones which indicate accelerated evolution and shows high intron dynamics in Drosophila lineage. As seen from Figure 2 only one intron (phase 2 intron in front of the $\mathrm{SH} 2$ domain) is common to all analyzed organisms. The position of the interrupted amino acid is always the same, just in front of WY/F, the conserved N-terminus of the SH2 domain. Interestingly, intron at the identical position is also present, not only in some other non-receptor PTK genes like $c s k$, $s r c$ and $f r k,{ }^{18}$ but also in many SH2 domains found in other unrelated genes. ${ }^{20}$ The SH2 domains in all these different, multidomain proteins must have originated from the same ancestral proto-SH2 domain and the common evolutionary heritage is still visible in their gene structures. Shuffling of the ancient, SH2 encoding gene to many unrelated genes happened very early in the evolution of animal multicellularity. ${ }^{18}$

The ancestral metazoan btkSD gene was intronrich. Position of introns in sponge $b t k S D$ gene probably reflects the archetype of the metazoan Tec family gene. From 15 introns within coding region most of them, 47 $\%$ were in phase 0 . Overabundance of phase 0 introns was observed in many species ${ }^{21}$ and this preference was probably present also in metazoan ancestor.

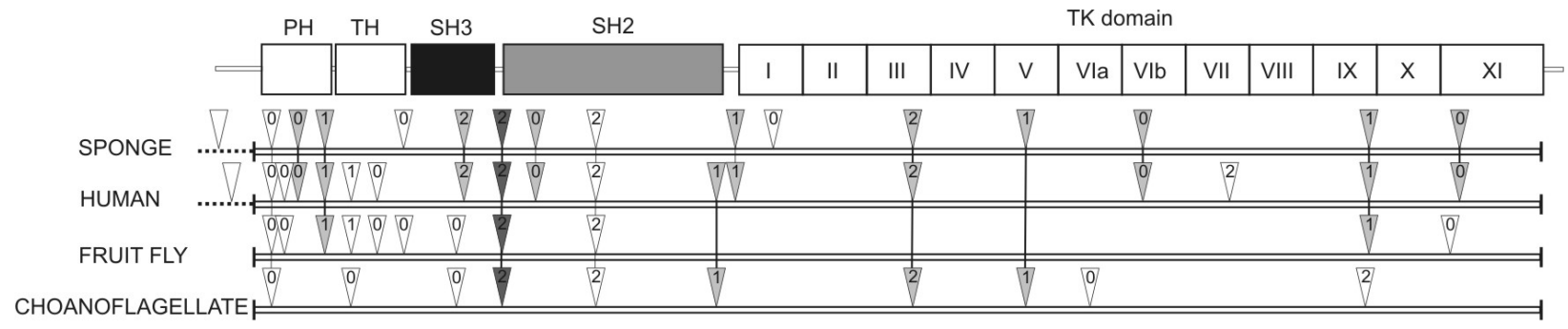

Figure 2. Schematic presentation of intron positions in btk PTK gene from sponge S. domuncula, human, fruit fly and choanoflagellate. Regions encoding $\mathrm{PH}, \mathrm{TH}, \mathrm{SH} 3, \mathrm{SH} 2$ and TK domains (divided in subdomains I-XI) are indicated. The positions of introns are marked by arrowheads and phases by numbers in arrowheads. Intron in front of SH2 domain (phase 2) is conserved in all genes and is shown with dark grey arrowhead. Introns shown with grey arrowheads are found at the same positions in at least 2 organisms and those shown with white arrowheads are gene-specific. Arrowheads connected with black line represent introns which are present at the same positions and phases. Arrowheads connected with thin line show introns which are in the same phase, but at slightly different positions (shifted for several aa). Sequences of genes (with indicated intron positions) were taken from the corresponding genomic databases. 
AP-1 A0 : CAGGGT CAACTGTCCTTGTTGCACGGCCAAACTGCTCAGAGAGAACCTEGCGAGTCATTTTCATGTGÄTGATGCTCCAGTCCTTAATAGATGAGGTGGTCTCTGAGATTGAACGGTTTG

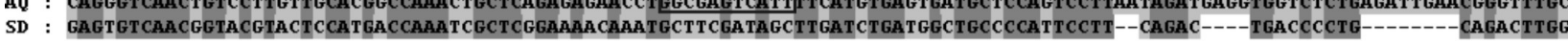

Pu box

AQ : TTCTTATGCTGTTTCGTTCCCTCTGGCCCAGACA CAAAGACAGGACCAGTGGCTCCCCCAGTAGGAGCAGTAGGAGCTCCTCCCCCTCOTTTTGAAGGAGGCTCATCTTGA--TCAGAATC

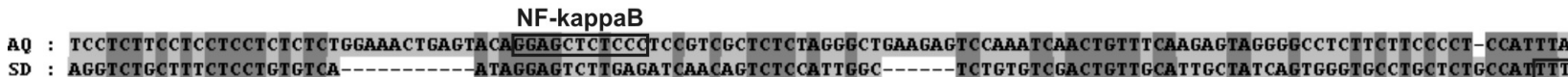

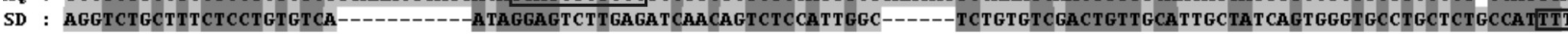

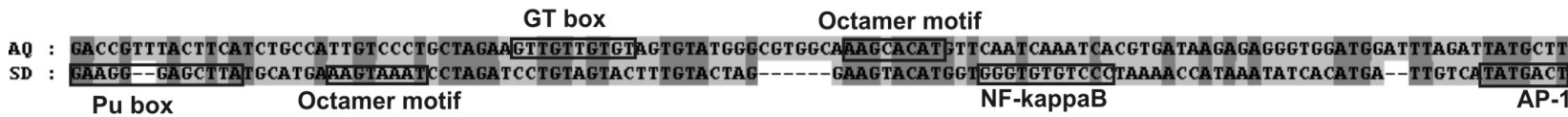

\section{aQ : TATta-Atcattagagätatacctcagagtatacaggantätattc}

SD : TATTCCGTTTCTTGATGACGTCATGTGTTTTGTCCAAGAaAGTTAGGT

GT box TTS

Figure 3. The structure of btk promoters from two sponges (S. domuncula and A. queenslandica). Putative motifs for binding of transcription factors and coactivators which have been demonstrated to bind to the human $b t k$ promoter were found and designated (Pu box, GT box, Octamer motif and NF-kappa B) in both sponges. Motif AP-1 was found in sponges only. TSS indicates transcription start site.

The promoter regions of $S$. domuncula and $A$. queenslandica btk genes were analyzed. Putative motifs for binding of transcription factors were found in both promoters. Pu box, GT box, Octamer motif and NFkappa B were identified in promoters of both sponges when non-stringent cut-offs were used (Figure 3). These transcription factors and coactivators have been demonstrated to bind to the human btk promoter. ${ }^{22}$ The structure of sponge $b t k$ promoter indicates similar gene regulation as in the human homolog. However, in both

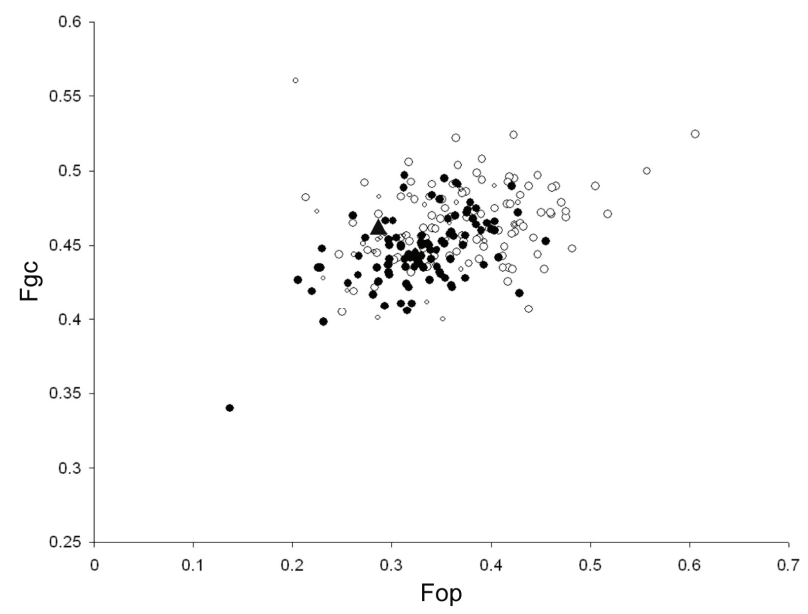

Figure 4. Fop plot of $224 \mathrm{~S}$. domuncula genes (frequency of optimal codons are ploted against $\mathrm{G}+\mathrm{C}$ content $-\mathrm{Fgc}$ ). Contigs and ESTs coding for ribosomal proteins are marked with $O$, singlets and cDNAs codings for the Ras family proteins are marked with $\bullet$. Remaining sequences are marked with $\circ$. Black triangle $(\boldsymbol{\Delta})$ indicates the position of BtkSD. Codon usage of gene $b t k S D$ indicates that this gene is relatively lowly expressed in sponge. sponges other common motifs were identified, not found in human, like AP-1 binding site (Figure 3). Interestingly, all of these motifs are not located at same positions in these two sponges which indicate fast evolving nature of sponge promoters. In accordance with this is observance that sponge $S$. domuncula lacks strong TATA-box signal in -60 bp region, while in $A$. queenslandica two TATA-box like motifs were identified (from -32 to $-23 \mathrm{bp}$ AATATACCTC and from -20 to -11 bp AGTATACAGG).

Investigation of the preferred codons in marine sponge $S$. domuncula revealed that highly expressed genes preferentially use C- and G-ending codons. ${ }^{17}$ Sponges as the oldest metazoan lineage probably provide the best possible insight into the characteristics of the genome of the metazoan ancestor. The preference for C- and G-ending codons has previously been documented in other metazoans. ${ }^{23-26}$ Therefore, it is likely that the codon usage bias was present in the metazoan ancestor. Fgc, GC3S and Fop for cDNA encoding BtkSD protein, were calculated using CODONW program. Fop parameters were based on results of correspondence analysis of $224 \mathrm{~S}$. domuncula genes. ${ }^{17} \mathrm{Fgc}$ for BtkSD was $46 \%$, Fop 0.287 and $\mathrm{G}+\mathrm{C}$ content at silent codon position $38.2 \%$. Fop varies from 13.7 to $60.6 \%$. Potentially highly and lowly expressed genes are distinguished in Figure 4 according to their Fop and $\mathrm{G}+\mathrm{C}$ content (Fgc). Conspicuously, ribosomal proteins and contigs are clustered more to the right, which indicates higher optimal codon usage. Codon usage in gene $b t k S D$ indicates that this gene is relatively lowly expressed in $S$. domuncula (Figure 4). This is further corroborated with the finding that there is only one BTK transcript in the $S$. domuncula EST library with over 13000 random sequences. 


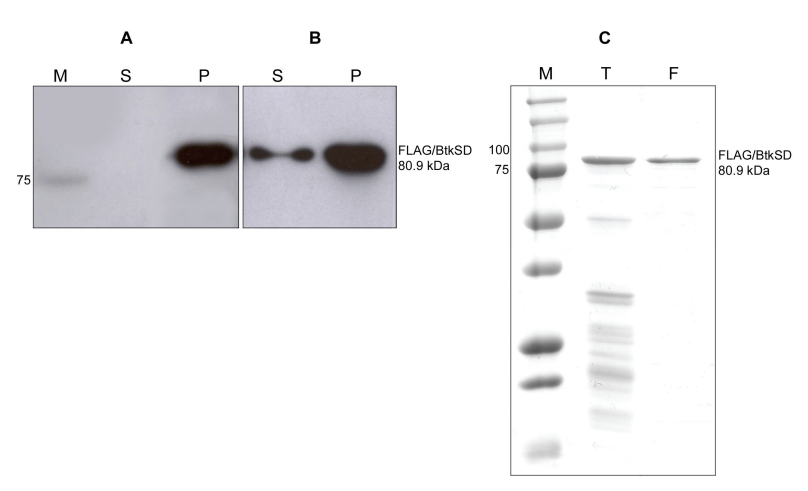

Figure 5. Purification of full-length BtkSD and Western blot analysis with anti-His antibodies. Under standard growth conditions (A) BtkSD could be detected in pellet (P), but not in supernatant $(\mathrm{S})$. Soluble protein was obtained under modified growth conditions $(\mathrm{B})$ : $\mathrm{OD}_{600} 1.5$ and decreased amount of IPTG $(0.05 \mathrm{mM})$ and then purified $(\mathrm{C})$ with TALON (T) and ion-exchange chromatography $(\mathrm{F})$.

\section{Protein BtkSD from Marine Sponge $S$. domuncula}

Recombinant protein is present only in pellet fraction under standard growth conditions (Figure 5A). Soluble recombinant protein was obtained under modified growth conditions as described in materials and methods section (Figure 5B). Coomassie Blue staining showed that after cobalt-charged agarose column (TALON) protein was $\sim 80 \%$ pure, and after Mono Q was $>90 \%$ pure (Figure $5 \mathrm{C}$ ). Kinase activity of purified BtkSD was tested as described in materials and methods section. Reaction was done in triplicate (Figure 6). As shown in Figure 6 the lag phase $\sim 120 \mathrm{~s}$ was pronounced as in human less phosphorylated Btk Mono Q fraction. ${ }^{27}$ Human Btk sample reached observed $V_{\max }$ of $174 \mathrm{~min}^{-1} .^{27}$ Our average observed $V_{\max }$ of $131 \mathrm{~min}^{-1}$ indicates very similar kinase activity of sponge ancestral Tec family member.

Our results indicate that this ancestral-type protein is probably structurally and functionally similar to the highly sophisticated multifunctional enzyme it is today, whose functions are usually associated with higher Metazoans. The biological role of Btk-like protein in the simple sponge organisms, lacking any organized tissue, is yet unknown, but it is possible that the basic role of Btk-like protein in sponges is associated with actin remodeling. In mammals, Btk is translocated in regions of membrane, lamellipodia, which are formed by the polymerization of actin. ${ }^{28}$ It has been shown that Btklike protein from Drosophila is required for controlling contraction of the actin-myosin microfilament ring during embryonic cellularization. ${ }^{29}$ Sponges are able to control the water current through their bodies by lowering the pressure in one region to draw water from another. Myocytes in sponges surround each osculum,

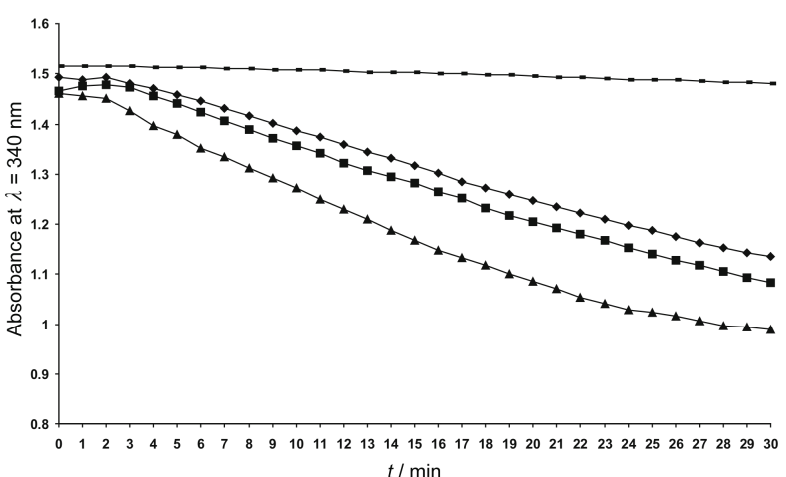

Figure 6. Kinase activity of BtkSD was determined by measuring the oxidation of NADH in a coupled pyruvate kinaselactate dehydrogenase enzyme assay system. Reactions were initiated by the addition of $25 \mathrm{nM}$ of BtkSD enzyme in $25 \mathrm{mM}$ HEPES $\mathrm{pH}=7.5,25 \mathrm{mM} \mathrm{NaCl}, 10 \mathrm{mM} \mathrm{KCl}, 10 \mathrm{mM} \mathrm{MgCl}_{2}$, $2 \mathrm{mM}$ phosphoenolpyruvate, $0.25 \mathrm{mM}$ NADH, $1 \mathrm{mM} \mathrm{ATP}, 2$ mM DTT, 2 units of pyruvate kinase, 3 units of lactate dehydrogenase, $0.008 \%$ triton X-100 and $0.2 \mathrm{mg} / \mathrm{mL}$ poly EY. Activity was monitored measuring the decrease in absorbance at $\lambda=340 \mathrm{~nm}$ for 30 minutes. Top line represents control reaction. Lower curves represent reaction in triplicate.

have actin and myosin and can contract. Changes in osculum pore size can also prevent the entry of unwanted material. ${ }^{30}$ Modulation of actin polymerization by Tec family kinases may have important role even in processes of cell proliferation, cell shape, motility and cell adhesion. ${ }^{28}$ The other possible function of Tec family ancestral-type protein in sponge is regulation of apoptosis. Apoptosis is general metazoan process for controlling tissue homeostasis in all developmental stages. Overexpression of Btk in HeLa cells led to apoptosis, but endogenous levels of Btk protected cells from apoptosis. ${ }^{31}$ Sponges possess homologs of genes involved in apoptotic pathways in human cells. ${ }^{32,33}$ However, molecular mechanism of apoptosis in sponges is yet unknown and Btk may play important role in deciding the fate of the cell depending on the biological response specific for the cell type.

\section{CONCLUSION}

In conclusion, this paper emphasizes four major points: (i) the sponge $b t k S D$ gene reflects the structural characteristics of the metazoan ancestral Tec family gene which was intron-rich; (ii) the structure of sponge promoter indicates similar gene regulation as in the human homolog; (iii) based on codon usage analysis and EST library search, btkSD gene is probably relatively lowly expressed in $S$. domuncula; (iv) the kinase activities of the sponge BtkSD enzyme and human homolog are similar. 
Therefore, we presume that even at the evolutionary stage of first metazoans this ancestral-type protein was structurally and functionally similar to the multifunctional enzyme it is today.

Acknowledgements. Authors would like to dedicate this work to the late Dr. Vera Gamulin. This work was funded by Croatian MSES grants 098-0982913-2478 (H. Ćetković). Professor WEG Müller is gratefully acknowledged for $S$. domuncula genomic and cDNA library.

\section{REFERENCES}

1. C. Ortutay, B. F. Nore, M. Vihinen, and C. I. Smith, Adv. Genet. 64 (2008) 51-80.

2. G. Manning, D. B. Whyte, R. Martinez, T. Hunter, and S Sudarsanam, Science 298 (2002) 1912-1934.

3. H. Ćetkovic, W. E. Muller, and V. Gamulin, Genomics 83 (2004) $743-745$.

4. C. I. Smith, T. C. Islam, P. T. Mattsson, A. J. Mohamed, B. F. Nore, and M. Vihinen, Bioessays 23 (2001) 436-446.

5. C. I. Smith, B. Baskin, P. Humire-Greiff, J. N. Zhou, P. G. Olsson, H. S. Maniar, P. Kjellen, J. D. Lambris, B. Christensson, L. Hammarstrom et al., J. Immunol. 152 (1994) 557-565.

6. A. B. Satterthwaite and O. N. Witte, Immunol. Rev. 175 (2000) 120-127.

7. M. E. Conley, A. K. Dobbs, D. M. Farmer, S. Kilic, K. Paris, S. Grigoriadou, E. Coustan-Smith, V. Howard, and D. Campana, Annu. Rev. Immunol. 27 (2009) 199-227.

8. D. J. Rawlings, D. C. Saffran, S. Tsukada, D. A. Largaespada, J. C. Grimaldi, L. Cohen, R. N. Mohr, J. F. Bazan, M. Howard, N. G. Copeland et al., Science 261 (1993) 358-361.

9. C. W. Li, J. Y. Chen, and T. E. Hua, Science 279 (1998) 879882 .

10. M. Harcet, M. Roller, H. Ćetkovic, D. Perina, M. Wiens, W. E. Muller, and K. Vlahoviček, Mol. Biol. Evol. 27 (2010) 27472756

11. M. Harcet, L. Lukić-Bilela, H. Ćetkovic, W. E. G. Muller, and V. Gamulin, Croat. Chem. Acta 78 (2005) 343-348.

12. M. Srivastava, O. Simakov, J. Chapman, B. Fahey, M. E. Gauthier, T. Mitros, G. S. Richards, C. Conaco, M. Dacre, U. Hellsten, C. Larroux, N. H. Putnam, M. Stanke, M. Adamska, A. Darling, S. M. Degnan, T. H. Oakley, D. C. Plachetzki, Y. Zhai, M. Adamski, A. Calcino, S. F. Cummins, D. M. Goodstein, C. Harris, D. J. Jackson, S. P. Leys, S. Shu, B. J. Woodcroft, M.
Vervoort, K. S. Kosik, G. Manning, B. M. Degnan, and D. S. Rokhsar, Nature 466 (2010) 720-726.

13. J. Seack, S. Perović, V. Gamulin, H. C. Schroder, P. Beutelmann, I. M. Muller, and W. E. Muller, Biochim. Biophys. Acta 1520 (2001) 21-34.

14. J. D. Thompson, T. J. Gibson, F. Plewniak, F. Jeanmougin, and D. G. Higgins, Nucleic Acids Res. 25 (1997) 4876-4882.

15. T. Heinemeyer, E. Wingender, I. Reuter, H. Hermjakob, A. E. Kel, O. V. Kel, E. V. Ignatieva, E. A. Ananko, O. A. Podkolodnaya, F. A. Kolpakov, N. L. Podkolodny, and N. A. Kolchanov, Nucleic Acids Res. 26 (1998) 362-367.

16. L. Milanesi, M. Muselli, and P. Arrigo, Comput. Appl. Biosci. 12 (1996) 399-404.

17. D. Perina, M. Harcet, A. Mikoč, K. Vlahoviček, W. E. G. Muller, and H. Ćetkovic, Food Technol. Biotechnol. 47 (2009) 269274.

18. H. Ćetkovic, V. A. Grebenjuk, W. E. Muller, and V. Gamulin, Gene 342 (2004) 251-261.

19. W. E. Muller, M. Bohm, V. A. Grebenjuk, A. Skorokhod, I. M. Muller, and V. Gamulin, Gene 295 (2002) 299-309.

20. C. M. Manning, W. R. Mathews, L. P. Fico, and J. R. Thackeray, Genetics 164 (2003) 433-442.

21. W. G. Qiu, N. Schisler, and A. Stoltzfus, Mol. Biol. Evol. 21 (2004) 1252-1263.

22. L. Yu, A. J. Mohamed, O. E. Simonson, L. Vargas, K. E. Blomberg, B. Bjorkstrand, H. J. Arteaga, B. F. Nore, and C. I. Smith, Blood 111 (2008) 4617-4626.

23. M. Gouy, and C. Gautier, Nucleic Acids Res. 10 (1982) 7055 7074 .

24. J. L. Bennetzen, and B. D. Hall, J. Biol. Chem. 257 (1982) 3026 3031 .

25. M. Mitreva, M. C. Wendl, J. Martin, T. Wylie, Y. Yin, A. Larson, J. Parkinson, R. H. Waterston, and J. P. McCarter, Genome Biol. 7 (2006) R75.1-R75.19.

26. H. Akashi, Genetics 136 (1994) 927-935.

27. L. Lin, R. Czerwinski, K. Kelleher, M. M. Siegel, P. Wu, R. Kriz, A. Aulabaugh, and M. Stahl, Biochemistry 48 (2009) 20212032.

28. L. D. Finkelstein and P. L. Schwartzberg, Trends Cell. Biol. 14 (2004) 443-451.

29. J. H. Thomas and E. Wieschaus, Development 131 (2004) $863-$ 871.

30. G. R. Elliott and S. P. Leys, J. Exp. Biol. 210 (2007) 3736-3748.

31. T. C. Islam and C. I. Smith, Immunol. Rev. 178 (2000) 49-63.

32. M. Wiens, B. Diehl-Seifert, and W. E. Muller, Cell Death Differ. 8 (2001) 887-898.

33. M. Wiens, A. Krasko, C. I. Muller, and W. E. Muller, J. Mol. Evol. 50 (2000) 520-531. 